\title{
Análisis bibliométrico de la producción científica sobre Riesgo psicosocial laboral, publicada entre 2000 y 2010*
}

\author{
Bibliometric analysis of scientific output on \\ psychosocial risk at work, 2000-2010
}

\begin{abstract}
Ninfa Pulido Moreno Universidad Pedagógica y Tecnológica de Colombia

Alexandra Puentes Suárez Universidad Pedagógica y Tecnológica de Colombia

Zaydi Luney Cruz Universidad El Bosque, Colombia

Diana Rocio López

Universidad El Bosque, Colombia

Mónica García Rubiano**

Universidad El Bosque, Colombia

Recibido: 15 de marzo de 2014 Revisado: 3 de mayo de 2014 Aceptado: 1 de julio de 2014
\end{abstract}

\section{Resumen}

El objetivo de este estudio fue describir la producción científica publicada sobre riesgo psicosocial laboral, en las bases de datos ProQuest y PsycArticles, durante los años 2000 a 2010. Para la recolección de información se elaboró una rejilla con indicadores bibliométricos, clasificados en tres categorías: bibliométricas, metodológicas, y teóricas de los artículos publicados. Se identificaron y analizaron 133 artículos científicos sobre riesgo psicosocial laboral, identificando que el año de mayor producción, fue el 2000 y 2005 , con 20 y 19 artículos publicados respectivamente, el $30,8 \%$ de los estudios se realizaron en Estados Unidos, la revista Journal of Occupational Health Psychology, reporta el mayor número de artículos $(18 \%)$, el $90,6 \%$ de los autores pertenecen a la categoría de transeúntes, el $91,7 \%$ de los artículos son de carácter empírico, con un diseño transeccional $(65,4 \%)$, los cuestionarios son los más utilizados para evaluar el riesgo (52,6\%), y el modelo teórico demanda control, es el más referido en las publicaciones $(39,8 \%)$.

Artículo de investigación realizado por docentes de la Universidad Pedagógica y Tecnológica de Colombia, Facultad de Ciencias de la Salud, Escuela de Psicología. Investigadoras del Grupo de Investigación Salud, Trabajo y Calidad de Vida.

Correspondencia: Mónica García Rubiano. Universidad El Bosque, Bogotá, Colombia. Dirección postal: carrera 9 \# 131A-02, edificio Fundadores, segundo piso. Correo electrónico: monica.garcia@gmail.com 
Palabras clave: factores psicosociales laborales, riesgo psicosocial, factor de riesgo psicosocial laboral, riesgo psicosocial en el trabajo, estudio bibliométrico.

\section{Abstract}

The aim of this study was to describe the scientific production published on psychosocial risk at work in the ProQuest and PsycArticles databases between 2000 and 2010. Data collection was assisted by a grid with bibliometric indicators in three different categories: bibliometric, methodological, and theoretical features of published articles. We identified and analyzed 133 scientific articles on psychosocial risk at work, with the most productive years being 2000 and 2005, with 20 and 19 articles respectively. $30.8 \%$ of the studies were conducted in the United States, the Journal of Occupational Health Psychology, reported the highest number of articles (18\%), $90.6 \%$ of the authors belong to the category of pedestrians, $91.7 \%$ of the items are of an empirical nature with a transactional design $(65,4 \%)$, questionnaires are most commonly used to assess risk $(52.6 \%)$, and the theoretical model of demand-control is the most referenced in publications (39.8\%).

Keywords: Psychosocial work factors, psychosocial risk factor of occupational psychosocial risk, psychosocial risk at work, bibliometric study.

\section{Introducción}

En la actualidad, el trabajo se concibe como un fenómeno psicosocial, resultado de la interacción de los individuos que lo llevan a cabo, en un marco social específico, y que además se encuentra influenciado por factores sociales, culturales, económicos, políticos e incluso legales (Peiró, Prieto y Roe, citados en Guillén y Guil, 2000; Barling, Kelloway e Iverson, 2003). En el ámbito laboral, todas las transformaciones que se ocasionan pueden ser una fuente generadora de riesgo para las personas, causando un deterioro significativo en su bienestar.

La Organización Internacional del Trabajo (OIT, 2010a), en el documento Riesgos emergentes y nuevos modelos de prevención en un mundo de trabajo en transformación, presenta los nuevos riesgos del trabajo que pueden ser provocados por la innovación técnica o por el cambio social u organizativo como: 1) las nuevas tecnologías y procesos de producción, por ejemplo, la nanotecnología o la biotecnología; 2) nuevas condiciones, como mayores cargas de trabajo, intensificación de las labores por recortes de plantilla, inadecuadas condiciones asociadas con la migración por motivos de trabajo en la economía informal; y 3) nuevas formas de empleo, como el empleo independiente, la subcontratación y los contratos temporales.

Al considerar el trabajo como un proceso de interacciones permanentes, es necesario abordar los factores de riesgo psicosocial dentro de una estructura específica, que afecta a los individuos en sus diferentes dimensiones (personal, familiar, laboral y social) y su propensión a reaccionar según ciertos patrones. Esta susceptibilidad está determinada por factores genéticos e influencias culturales y ambientales; al producirse un desajuste en uno de estos, el organismo reacciona de manera específica, a través de respuestas fisiológicas, emocionales y comportamentales (Oliver, Cheyne, Thomas y Cox, 2002; Bennett, Lehman y Reynolds, 2000).

La existencia de factores sociales y exigencias psicológicas en el ejercicio de las profesiones u oficios generan reacciones fisiológicas que, sumadas al bajo control que el individuo puede tener sobre las exigencias y demandas del contexto, incrementan la vulnerabilidad del trabajador ante los factores de riesgo. La interrelación negativa entre las condiciones laborales (medio ambiente de trabajo, tareas y características de la organización laboral) y los factores individuales (capacidades, necesidades y expectativas), entre otros, originan consecuencias nocivas en el desempeño, la satis- 
facción y la salud de las personas (Jutinico, 1996; Bond y Bunce, 2001).

Según Peiró (2004), los diferentes cambios en el ámbito laboral causan un deterioro significativo de la salud y el bienestar del trabajador, por lo tanto es de vital importancia desarrollar estudios donde se haga un análisis preciso de los factores de riesgo psicosocial, con el fin de fortalecer las políticas nacionales e institucionales que garanticen el desarrollo de intervenciones dirigidas a prevenir, neutralizar y amortiguar efectos negativos de los riesgos psicosociales, los cuales, a largo plazo, traen consecuencias aún más nocivas que las producidas por los riesgos abordados desde la higiene y la seguridad en el trabajo (Bond, Punnett, Pyle, Cazeca y Cooperman, 2004).

Planteamientos como los de Seabury, Lakdawalla y Reville ( citados en Andrade y Gómez, 2008) quienes refieren que se deben promover entornos de trabajo cada vez más saludables, que propicien la calidad de vida y el bienestar de las personasevidencian la necesidad de fortalecer investigaciones orientadas a la evaluación e identificación de los factores de riesgo en el contexto laboral y la influencia de estos sobre las diferentes esferas de la vida del individuo, así como la importancia de contar con definiciones claras y teóricamente sustentadas (Bültmann, Kant,Van Den Brandt y Kasl, 2002; Cavanaugh, Boswell, Roehling y Boudreau, 2000; Day y Jreige, 2002).

En Colombia, el Ministerio de la Protección Social (2008), a través de la Resolución 2646 de 2008, establece disposiciones y responsabilidades que van desde la identificación hasta la intervención y monitoreo de los riesgos psicosociales. En su artículo 3 , define los factores de riesgo psicosocial como todas aquellas condiciones psicosociales cuya identificación y evaluación muestran efectos negativos en la salud de los trabajadores o en el trabajo; además, considera como fuentes de riesgo los factores intralaborales, extralaborales e individuales.

Asimismo, en el capítulo Iv, refiere la importancia de acudir a las disposiciones de los organismos internacionales para determinar las patologías derivadas del estrés, además de acudir a los centros de investigación cuyos estudios documenten y evidencien la relación de causalidad a partir de publicaciones científicas, ya sea en el país o en el ámbito internacional.

Desde dicha perspectiva, este estudio permite identificar la producción bibliográfica existente sobre los riesgos psicosociales en el ámbito laboral, desarrollada entre los años 2000 y 2010, con el fin de reconocer teorías, metodologías utilizadas, poblaciones objeto de estudio, características y variables de los instrumentos y técnicas de evaluación. De esta manera, permite determinar, desde los indicadores bibliométricos definidos para el estudio, el estado de la investigación en el tema y ser un referente para formular a futuro proyectos de investigación desde la psicología ocupacional, así como la revisión y consolidación de modelos teóricos que permitan abordar los factores de riesgo laboral, desde la prevención, promoción e intervención.

\section{Objetivo}

Describir a través de indicadores bibliométricos la producción científica relacionada con riesgo psicosocial laboral, en las bases de datos ProQuest y PsycArticles, durante el periodo 2000-2010.

\section{Método}

Investigación cuantitativa de tipo descriptivo. Para el análisis de la información se utilizó la bibliometría, que permite el análisis cuantitativo de la producción científica a través de la literatura, estudiando la naturaleza y el curso de una disciplina científica, los cuales tienen por objeto el tratamiento y análisis cuantitativo de las publicaciones científicas (López, citado en Camps \& Chauhan, 2009). Con relación a los indicadores bibliométricos, los parámetros se usan para determinar la productividad de los autores o instituciones, medida por 1) el número de sus trabajos, 2) el crecimiento de cualquier campo de la ciencia, 3) la variación cronológica del número de trabajos publicados, 4) la colaboración entre los científicos o instituciones, 5) el número de autores por trabajo o centros de investigación que colaboran. (Sancho, 1990). 


\section{Unidades de análisis}

Se identificaron y analizaron 133 artículos científicos sobre riesgo psicosocial laboral, publicados en las bases de datos ProQuest y PsycArticles, en el periodo 2000-2010. Para determinar los artículos por ser analizados, se tuvieron en cuenta los siguientes requisitos: 1) artículos cuyo texto completo fuera accequible a las investigadoras; 2) artículos publicados en las bases de datos ProQuest y PsycArticles, correspondientes al periodo 2000 2010; 3) una temática de estudio sobre factores psicosociales desarrollada en contextos de trabajo; y 4) artículos identificados a partir del uso de los descriptores, definidos para este estudio.

La búsqueda de la información se realizó teniendo en cuenta los siguientes descriptores: 1) psychosocial labor risk; 2) psychosocial risk factors work; 3) psychosocial work risks; 4) psychosocial risk factor job, 5) psychosocial risk, y 6) psychosocial hazard.

\section{Instrumento}

El instrumento se adaptó a las necesidades de la investigación y se ajustó siguiendo los criterios propuestos por Meneses, Calle y García (2008). Finalmente se obtuvo una rejilla con indicadores bibliométricos, definidos para el estudio. Los indicadores se dividieron en tres características: bibliométricas, metodológicas y teóricas de los artículos publicados.

Características bibliométricas. Publicaciones por año, por bases de datos, por país donde se realiza el estudio; publicaciones de acuerdo al número de páginas, y según el número de referencias reportadas; publicaciones por nombre de la revista, por autor, por la afiliación de los autores; y artículos por número de firmas.

Características metodológicas. Publicaciones por tipo de documento, según el enfoque de investigación; publicaciones de acuerdo con el tipo de estudio, según el diseño de investigación; por número de participantes que constituyeron la muestra; publicaciones según el género de los participantes; publicaciones por tipo de muestreo; publicaciones según el tratamiento estadístico de los datos; y publicaciones por técnica e instrumentos utilizados para hacer la recolección de la información.

Características teóricas. Publicaciones según el modelo teórico, y por tipo de riesgo psicosocial laboral.

\section{Procedimiento}

En primer lugar se determinaron los descriptores, se identificaron y definieron los indicadores bibliométricos, y se adaptó la rejilla de registro, para sistematizar la información. Luego se realizó la búsqueda de la producción científica en las bases de datos ProQuest y PsycArticles, para el periodo 2000-2010, y se identificaron las unidades de análisis por tener en cuenta en el estudio.

Finalmente se realizó la revisión y análisis de cada uno de los artículos encontrados por parte de las investigadoras, la consolidación de la información en la base de datos diseñada para el estudio en el paquete estadístico SPSS, la tabulación y análisis de datos, teniendo en cuenta las características bibliométricas, metodológicas y teóricas.

Este estudio se realizó teniendo en cuenta los lineamientos de la Resolución 008430 del 4 de octubre de 1993, del Ministerio de Salud, por la cual se establecen las normas científicas, técnicas y administrativas para la investigación en salud. La presente investigación se clasifica en la categoría de investigación sin riesgo, en la cual no se realiza ninguna intervención o modificación intencionada de las variables biológicas, fisiológicas, psicológicas o sociales de los individuos que participan en el estudio.

De igual forma, de acuerdo con el Manual deontológico y bioético del psicólogo, Ley 1090 de 2006, en su capítulo vil, se es responsable de los temas de estudio, metodología y materiales de la investigación, así como del análisis de resultados, conclusiones y divulgación. En el desarrollo de este estudio bibliométrico, se hizo un análisis cuidadoso del contenido de la producción científica consultada, manteniendo la veracidad de la 
información y garantizando los derechos de autor de las fuentes revisadas.

\section{Resultados}

Los resultados del análisis bibliométrico se presentan teniendo en cuenta las características bibliométricas, metodológicas y teóricas, de los 133 artículos sobre Riesgo Psicosocial Laboral tomados como unidades de análisis.

\section{Características bibliométricas de los artículos publicados}

El mayor número de artículos se presentó en 2000 (15\%). El 62,4\% de los artículos se encuentran en la base de datos PsycArticles. En Estados Unidos se han realizado la mayoría de los estudios (30,8\%); el $39 \%$ de los artículos tienen entre 6 y 10 páginas, y el $37,6 \%$ de los artículos reportan entre 31 y 45 referencias (tabla 1 ).

Tabla 1.

Indicadores bibliométricos

\begin{tabular}{|c|c|c|c|}
\hline Indicador & Categoría & Frecuencia & Porcentaje \\
\hline \multirow{11}{*}{ Año de publicación } & 2000 & 20 & 15,0 \\
\hline & 2005 & 19 & 14,3 \\
\hline & 2001 & 17 & 12,8 \\
\hline & 2002 & 16 & 12,0 \\
\hline & 2006 & 12 & 9,0 \\
\hline & 2004 & 11 & 8,3 \\
\hline & 2003 & 10 & 7,5 \\
\hline & 2007 & 9 & 6,8 \\
\hline & 2008 & 9 & 6,8 \\
\hline & 2009 & 8 & 6,0 \\
\hline & 2010 & 2 & 1,5 \\
\hline \multirow{3}{*}{ Base de datos } & PsycArticles & 83 & 62,4 \\
\hline & ProQuest & 46 & 34,6 \\
\hline & ProQuest y PsycArticles & 4 & 3,0 \\
\hline \multirow{17}{*}{ País donde se realizó el estudio } & Estados Unidos & 41 & 30,8 \\
\hline & España & 11 & 8,3 \\
\hline & Finlandia & 10 & 7,5 \\
\hline & Canadá & 10 & 7,5 \\
\hline & Suecia & 7 & 5,3 \\
\hline & Inglaterra & 7 & 5,3 \\
\hline & Holanda & 7 & 5,3 \\
\hline & Australia & 7 & 5,3 \\
\hline & Noruega & 5 & 3,8 \\
\hline & Reino Unido & 4 & 3,0 \\
\hline & Dinamarca & 4 & 3,0 \\
\hline & Suiza & 3 & 2,2 \\
\hline & Francia & 3 & 2,2 \\
\hline & Hong Kong & 2 & 1,5 \\
\hline & Alemania & 2 & 1,5 \\
\hline & No refiere & 2 & 1,5 \\
\hline & Otros países* & 8 & 6,0 \\
\hline \multirow{5}{*}{ Número de páginas } & 6 a 10 & 52 & 39,0 \\
\hline & 11 a 15 & 50 & 37,6 \\
\hline & 16 a 20 & 21 & 15,8 \\
\hline & 1 a 5 & 5 & 3,8 \\
\hline & Más de 21 & 5 & 3,8 \\
\hline
\end{tabular}




\begin{tabular}{llll}
\hline Indicador & Categoría & Frecuencia & Porcentaje \\
\hline & 31 a 45 & 50 & 37,6 \\
& 46 a 60 & 30 & 22,6 \\
Número de referencias & 16 a 30 & 19 & 14,3 \\
& 61 a 74 & 18 & 13,5 \\
& Más de 75 & 14 & 10,5 \\
& 1 a 15 & 2 & 1,5 \\
\hline
\end{tabular}

*Países Bajos, Israel, Japón, Italia, Irlanda, Turquia, Sudan y Colombia.

Fuente:

Con relación a las publicaciones, $18 \%$ de los artículos revisados se encuentran publicados en el Journal of Occupational Health Psychology; el 9,6\%, en el Journal of Applied Psychology; y el 6,0\%, en
International Archives of Occupational and Environmental Health. Las demás revistas tienen un menor índice de publicación sobre la temática de riesgos psicosociales laborales (tabla 2).

Tabla 2.

Nombre de la publicación

\begin{tabular}{lcc}
\hline Nombre de la fuente & Frecuencia & Porcentaje \\
\hline Otras publicaciones & 49 & 37 \\
Journal of Occupational Health Psychology & 24 & 18 \\
Journal of Applied Psychology & 13 & 9,6 \\
International Archives of Occupational and Environmental Health & 8 & 6,0 \\
Journal of Occupational Rehabilitation & 5 & 3,7 \\
American Journal of Public Health & 5 & 3,7 \\
Journal of Epidemiology and Community Health & 4 & 3,0 \\
Scandinavian Journal of Work, Environment \& Health & 3 & 2,3 \\
International Journal of Epidemiology & 3 & 2,3 \\
Health Psychology & 3 & 2,3 \\
Scand J Public Health & 3 & 2,3 \\
Journal of Occupational and Environmental Medicine & 3 & 2,3 \\
Journal of Family Psychology & 2 & 1,5 \\
International Journal of Stress Management & 2 & 1,5 \\
Bio Med Central & 2 & 1,5 \\
Chronobiology International - informs healthcare & 2 & 1,5 \\
Journal of Advanced Nursing & 2 & 1,5 \\
\hline
\end{tabular}

Fuente: elaboración propia

En la tabla 3 se evidencia que el $90,6 \%$ de los autores que publicaron sobre el tema pertenece a la categoría de transeúntes; el $91 \%$, a instituciones universitarias; el $48,1 \%$ de los artículos fueron escritos entre 4 y 6 autores; y el 47,4\%, entre 1 y 3 autores.
Se encuentra un autor en la categoría de productores moderados (autores que han publicado entre 5 y 9 artículos), Mika Kivimaki, de la Universidad de Helsinki; 12, en la categoría de aspirantes (autores que tienen entre 2 y 4 publicaciones); y 226 
Tabla 3.

Información de los autores

\begin{tabular}{llcc}
\hline Indicador & Categoría & Frecuencia & Porcentaje \\
\hline \multirow{4}{*}{ Autores } & Transeúntes & 270 & 90,6 \\
& Aspirantes & 27 & 9,06 \\
& Productores moderados & 1 & 0,34 \\
\hline \multirow{4}{*}{ Afiliación de los autores } & Universidades & 121 & 91 \\
& Organismos gubernamentales & 2 & 1,5 \\
& Otro & 9 & 6,8 \\
& No refiere & 1 & 0,7 \\
\hline \multirow{3}{*}{ Número de firmas } & 4 a 6 autores & 64 & 48,1 \\
& 1 a 3 autores & 63 & 47,4 \\
& 7 o más autores & 6 & 4,5 \\
\hline
\end{tabular}

Fuente: elaboración propia

autores, en la categoría de transeúntes (autores con 1 publicación). No se encontraron autores en la categoría de grandes productores (autores con 10 o más publicaciones).

\section{Características metodológicas de los artículos publicados}

La tabla 4 evidencia un mayor porcentaje de artículos de carácter empírico (91,7\%), y el enfoque de investigación más reportado fue el cuantitativo $(88,0 \%)$. Los tipos de estudio más utilizados en los artículos revisados fueron el correlacional, con un $46,6 \%$, y los descriptivos, con un 31,6\%. El diseño de investigación mencionado en mayor porcentaje fue el transeccional o transversal, con un $65,4 \%$.

El 25,6\% de los artículos reportan entre 1001 y 5000 participantes, y se destaca que ningún artículo de los analizados toma menos de 30 sujetos de estudio. En los artículos revisados se identificó que se incluyeron tanto hombres como mujeres, en un $72,9 \%$.

En los 133 artículos revisados, el muestreo reportado con mayor frecuencia fue el muestreo no probabilístico o dirigido (89 artículos), y en 77 de los artículos se utilizó la estadística descriptiva e inferencial.

Tabla 4.

Indicadores metodológicos de los artículos

\begin{tabular}{llcc}
\hline Indicador & Categoría & Frecuencia & Porcentaje \\
\hline \multirow{2}{*}{ Tipo de documento } & Artículos empíricos & 122 & 91,7 \\
& Artículos teóricos & 11 & 8,3 \\
\hline \multirow{4}{*}{ Enfoque de investigación } & Cuantitativos & 117 & 88,0 \\
& No aplica & 11 & 8,2 \\
& Mixtos & 2 & 1,5 \\
& Cualitativos & 3 & 2,3 \\
\hline \multirow{5}{*}{ Tipo de estudio } & Correlacional & 62 & 46,6 \\
& Descriptivo & 42 & 31,6 \\
& No aplica & 11 & 8,3 \\
& Explicativo & 8 & 6,0 \\
& Exploratorio & 8 & 6,0 \\
& Explicativo - IAP & 2 & 1,5 \\
\hline
\end{tabular}




\begin{tabular}{|c|c|c|c|}
\hline Indicador & Categoría & Frecuencia & Porcentaje \\
\hline \multirow{5}{*}{ Diseño de investigación } & Transeccional o transversal & 87 & 65,4 \\
\hline & Longitudinal o evolutivo & 23 & 17,3 \\
\hline & No aplica & 11 & 8,3 \\
\hline & Preexperimental & 7 & 5,3 \\
\hline & Cuasi experimental & 5 & 3,7 \\
\hline \multirow{8}{*}{ Número de participantes } & 1001 a 5000 & 34 & 25,6 \\
\hline & 501 a 1000 & 19 & 14,3 \\
\hline & 101 a 200 & 18 & 13,5 \\
\hline & 201 a 300 & 18 & 13,5 \\
\hline & Más de 5000 & 15 & 11,3 \\
\hline & 301 a 500 & 13 & 9,8 \\
\hline & No aplica & 11 & 8,3 \\
\hline & 31 a 100 & 5 & 3,7 \\
\hline \multirow{5}{*}{ Género de los participantes } & Mixto & 97 & 72,9 \\
\hline & Mujeres & 13 & 9,8 \\
\hline & No aplica & 11 & 8,3 \\
\hline & No refiere & 7 & 5,3 \\
\hline & Hombres & 5 & 3,7 \\
\hline \multirow{5}{*}{ Tipo de muestreo } & Muestreo no probabilístico o dirigido & 89 & 66,9 \\
\hline & No refiere & 17 & 12,8 \\
\hline & No aplica & 11 & 8,3 \\
\hline & Muestreo aleatorio simple & 9 & 6,7 \\
\hline & Muestreo probabilístico estratificado & 7 & 5,3 \\
\hline \multirow{4}{*}{ Tratamiento estadístico } & Estadística descriptiva e inferencial & 77 & 57,9 \\
\hline & Estadística descriptiva & 30 & 22,5 \\
\hline & Estadística inferencial & 15 & 11,3 \\
\hline & No aplica & 11 & 8,3 \\
\hline
\end{tabular}

Fuente: elaboración propia

Los instrumentos más utilizados para realizar medición de riesgos psicosociales laborales, referidos en la unidades de análisis, son los cuestionarios $(52,6 \%)$, y en menor porcentaje las pruebas psico- fisiológicas (13,7\%), las entrevistas en el $10,2 \%$ y las encuestas en el $5,6 \%$ de los artículos. Se identificaron otros instrumentos y técnicas, en menor porcentaje (Tabla 5).

Tabla 5.

Distribución de instrumentos referidos en los artículos

\begin{tabular}{lcc}
\hline Instrumentos & Frecuencia & Porcentaje \\
\hline Cuestionarios & 93 & 52,6 \\
Pruebas psicofisiológicas & 24 & 13,7 \\
Entrevistas & 18 & 10,2 \\
No aplica & 11 & 6,2 \\
Encuestas & 10 & 5,6 \\
Pruebas psicológicas & 5 & 2,8 \\
Registros de observación & 3 & 1,7 \\
Listas de verificación & 3 & 1,7 \\
Pruebas fisiológicas & 2 & 1,1 \\
Pruebas de laboratorio & 2 & 1,1 \\
Inventarios & 2 & 1,1 \\
Escalas & 2 & 1,1 \\
Revisión de archivos & 2 & 1,1 \\
\hline Fuente & &
\end{tabular}

Fuente: elaboración propia 


\section{Características teóricas de los artículos publicados}

En la tabla 6 se observa que el modelo teórico demanda-control es el más utilizado, pues se evidencia en el $39,8 \%$ de los artículos, mientras que el modelo desequilibrio-esfuerzo-recompensa se retoma en el 6,0\% de los artículos. Por su parte, el $39,1 \%$ de los artículos no refieren ningún modelo teórico. En menor porcentaje, se encuentran otros modelos teóricos: el modelo de dimensiones de Warr; el modelo de Cohen, Casel y Walsh, Davidson Bergman Hallbera, Cooper y Kanter, Cooper y Marshall, el modelo de Loewen y Suedfeld; el modelo teórico de Frone relación ocupación y accidentalidad; y el modelo estructural de Kahn.

Tabla 6.

Modelos teóricos mencionados en los artículos

\begin{tabular}{lcc}
\hline Nombre del modelo & Frecuencia & Porcentaje \\
\hline Modelo demanda-control & 53 & 39,8 \\
\hline No refiere & 52 & 39,1 \\
\hline Otros modelos & 13 & 9,8 \\
\hline Modelo de desequilibrio-esfuerzo-recompensa & 8 & 6,0 \\
\hline Modelo demanda-control y modelo de apoyo social de Johnson & 3 & 2,3 \\
\hline Modelo de dos dimensiones de Warr & 2 & 1,5 \\
\hline Modelo de Lazarus & 2 & 1,5 \\
\hline
\end{tabular}

Fuente: elaboración propia

De acuerdo con la tabla 7, los factores de riesgo individual fueron reportados en el $91,7 \%$ de los ar- tículos; los factores de riesgo intralaboral, en el $85,7 \%$; y los factores extralaborales, en el $18,8 \%$.

Tabla 7.

Porcentaje de factores de riesgo psicosocial mencionados en los artículos

\begin{tabular}{lcc}
\hline Factor de riesgo psicosocial & Frecuencia & Porcentaje \\
\hline Factores de riesgo intralaboral & 114 & 85.7 \\
Factores de riesgo extra laboral & 25 & 18,8 \\
Factores de riesgo individual & 122 & 91,7 \\
\hline
\end{tabular}

Fuente: elaboración propia

Los factores de riesgo intralaboral más mencionados en los estudios son: control del trabajo (uso de habilidades-toma de decisiones), demandas de trabajo, estrés laboral percibido, soporte social, clima laboral, condiciones del medio ambiente de trabajo, falta de capacitación y entrenamiento, exigencias de responsabilidad del cargo, sobrecarga laboral cualitativa, factores ergonómicos, tensión laboral, estabilidad laboral, justicia organizacional y exigencias cuantitativas de la tarea.

Los factores de riesgo intralaboral menos referenciados son: tamaño de la organización, reconoci- miento y compensación, violencia por atención a personal, inequidad laboral (género), cada uno mencionado en dos artículos. Por su parte, los factores valoración del soporte organizacional, soporte social por parte de los subordinados, antigüedad en el trabajo, desempeño en el trabajo, gestión organizacional y estilo de dirección se refieren en solo un artículo.

Los riesgos psicosociales extralaborales reportados con mayor frecuencia en los artículos fueron: soporte social por parte de familiares, influencia del grupo familiar, situación económica del grupo 
familiar, composición familiar y relaciones familiares. Por su parte, entre los menos mencionados están: tiempo de desplazamiento, doble rol, poca participación social, vivir solo, trabajo en casa y nivel educativo del grupo social.

Los factores de riesgo individual más referidos en los artículos son: factores sociodemográficos, condiciones de salud, estilos de vida, rasgo de personalidad y salud mental; y los menos mencionados, autonomía y autorrealización.

\section{Discusión}

La investigación de los factores de riesgo psicosociales inherentes a los diferentes ámbitos de desempeño permite la comprensión del desarrollo de este campo de conocimiento, así como la identificación y análisis de las características bibliométricas de los artículos publicados, las características metodológicas, y las características conceptuales y teóricas de la producción científica, en las bases de datos ProQuest y PsycArticles, en el periodo 2000-2010, lo cual evidencia el desarrollo de esta temática y la relevancia para tomar decisiones a escala de investigación.

Respecto a las bases de datos, el $62,4 \%$ de los artículos se encuentran en PsycArticles y el 34,6\%, en ProQuest, porcentajes que permiten referir que puede existir una relación marcada entre el origen de las bases de datos, las revistas que en ellas se publican y los países en los cuales más se ha investigado sobre riesgo psicosocial laboral -es decir, países como Estados Unidos, Canadá e Inglaterra, entre otros-.

Con relación al número de páginas y de referencias, se encuentra que la mayoría de los artículos cuentan con más de 6 páginas y con más de 31 referencias. La facilidad para acceder a grandes bases de datos bibliográficas ha llevado a que muchos autores sean propensos a incluir extensas listas de referencias bibliográficas, que en ocasiones no han sido leídas en su totalidad (López, Ruíz y Jiménez, 2006). Por lo tanto, y tal como lo plantean estos mismos autores, cada revista debe contar con políticas editoriales que les permitan establecer límites en cuanto al número de referencias por incluir y al número de páginas.
Según López et al. (2006), las revistas científicas son un canal de comunicación científica, donde profesionales e investigadores de diferentes disciplinas pueden dar a conocer sus hallazgos o estar informados sobre nuevos avances y descubrimientos, e intercambiar experiencias. Para efectos de este estudio bibliométrico, se encuentran dos revistas que publican el $27,6 \%$ de los artículos sobre riesgo psicosocial laboral: el Journal of Occupational Health Psychology y el Journal of Applied Psychology.

Estas revistas tienen como una de sus directrices la publicación de temáticas relacionadas con la psicología ocupacional, área que es catalogada como un campo de conocimiento interdisciplinario, que busca el mejoramiento de la calidad de vida laboral y la protección y promoción de la seguridad, la salud y el bienestar de los trabajadores. La mayoría de los artículos se encuentran en publicaciones de medicina, epidemiología, salud pública y psicología, lo cual evidencia que el estudio de los riesgos psicosociales no ha sido un tema particular de la psicología; por el contrario, ha sido estudiado por la medicina en cuanto a su relación con enfermedades crónicas como la hipertensión arterial, enfermedad coronaria, entre otras.

La tendencia a publicar está dada por autores con una única publicación $(90,6 \%)$, mientras que en menor porcentaje se encuentran artículos escritos por dos a cuatro autores. En la categoría de grandes productores solamente se encuentra el psicólogo Mika Kivimaki, quien pertenece al grupo de investigación Personalidad y Bienestar, de la Universidad de Helsinki. Este grupo tiene como ejes de investigación el estrés, el estrés en el trabajo, y la comprensión de los factores de riesgo y de las enfermedades crónicas de relevancia para la salud pública.

Desde el punto de vista de la afiliación de los autores, se encuentra que el $91 \%$, está vinculados a una universidad; es decir que posiblemente muchos de los artículos científicos publicados son producto de investigaciones que se hacen desde los grupos de investigación o desde las investigaciones lideradas por institutos de salud o epidemiologia, dependientes de universidades. De igual forma, en los artículos se evidencia que en 
algunos casos los autores son de diferentes nacionalidades o centros de trabajo, lo cual indica la colaboración que existe entre autores y el fortalecimiento de la investigación y las publicaciones en esta área temática, indispensables en el desarrollo científico.

Para el análisis de las características metodológicas, en la mayoría de los artículos se observa que el investigador busca establecer el grado de relación existente entre dos o más variables, lo cual resulta pertinente al tener en cuenta que el riesgo psicosocial es un concepto que implica multicausalidad y lo componen diversas variables, entre ellas las mencionadas por Villalobos (2004), quien afirma que bajo determinadas circunstancias de intensidad y tiempo de exposición se generan efectos negativos en el trabajador o trabajadores, en la organización y en los grupos.

El propósito de la mayoría de investigaciones revisadas fue determinar cómo puede una variable ser influenciada por otra, además de los efectos percibidos por el individuo o por la organización, los cuales se reflejan en la escogencia de la propuesta de intervención según el caso. Cabe destacar el hecho de los pocos estudios explicativos, evidenciando el estancamiento en cuanto a nuevos modelos teóricos que puedan soportar la temática abordada.

De los artículos revisados, el $91,7 \%$ utilizaron el enfoque cuantitativo. Según Melía, Nogareda, Duro, Peiró, Salanova y Gracia (2006), las metodologías cuantitativas presentan como principales ventajas sus sólidos fundamentos en fiabilidad, validez, estandarización y baremación, y permiten en empresas de gran tamaño tener una representatividad de la información recolectada.

Se evidencian solo tres artículos desde el enfoque cualitativo, que se fundamentan en una perspectiva interpretativa, centrada en el entendimiento del significado de las acciones de los trabajadores en el contexto laboral; estos permiten captar elementos idiosincrásicos de la organización, analizar incidentes críticos y profundizar en aspectos específicos de interés, en este caso, sobre los factores de riesgo psicosocial laboral (Patton, citado en Melía et al., 2006).
En relación con la forma como se evalúan los riesgos psicosociales, se evidencia que los cuestionarios son los instrumentos más reportados en las publicaciones $(52,6 \%)$. Es posible que su utilización se deba a la facilidad en los procesos de recolección de información y de sistematización de aquella, lo cual puede relacionarse con el porcentaje de sujetos que participaron en las investigaciones, pues la mayoría de los artículos refieren muestras de más de 500 individuos (51,2\%). Con muestras tan grandes, el cuestionario parece una alternativa práctica, pero surgen cuestionamientos acerca de la confiabilidad y validez de estos instrumentos, aspectos no referidos en la mayoría de las unidades de análisis revisadas.

Adicionalmente se observa la utilización de otras técnicas e instrumentos; el 13,7\% reporta pruebas psicofisiológicas; el 10,2\%, entrevistas; y el 17,3\%, otros instrumentos. La información obtenida a través de los diferentes métodos se analiza y jerarquiza considerando aspectos como la gravedad, es decir, el impacto que genera en la población, sea por el número de personas afectadas o por la severidad de los efectos en la salud, y la susceptibilidad de cambio, relacionada con la posibilidad de efectuar ajustes en las condiciones de trabajo, que disminuyan los niveles y la nocividad del factor de riesgo o sus efectos (Villalobos, 2007). Igualmente, utilizar diferentes herramientas diagnósticas permite detectar niveles de discapacidad laboral y absentismo, asociados la presencia de riesgos psicosociales (Schaufeli y Salanova, 2002).

Con relación a las características teóricas de los artículos publicados, y desde el punto de vista de los modelos teóricos que se plantean en los diferentes artículos, se observa que el 39,8\% refiere el modelo teórico de demanda-control, y el 2,3\% retoma este modelo y consideran la variable apoyo social, planteada por Jhonson y Hall (1988). Según el Ministerio de Trabajo y Asuntos Sociales de España (s. f.), el modelo demanda-control-apoyo social fue desarrollado para describir y analizar situaciones laborales en las que los estresores son crónicos, y pone totalmente el acento en las características psicosociales del entorno de trabajo. $\mathrm{Ha}$ sido el modelo más influyente en la investigación sobre el entorno psicosocial de trabajo, estrés y 
enfermedad desde principios de los años ochenta, así como el que presenta mayor evidencia científica a la hora de explicar efectos en la salud.

Este modelo teórico se destaca por las evidencias empíricas que lo respaldan; el modelo de tensión laboral o demanda-control de Karasek ha sido utilizado en investigaciones, en su gran mayoría en países desarrollados (Estados Unidos y algunos países europeos), por lo que se necesitan más estudios representativos con estos modelos en Latinoamérica, pues siguen siendo insuficientes. En Colombia existen algunos estudios con el modelo demanda-control en diferentes poblaciones, que demuestran su validez y la relación con indicadores de salud (Gómez, 2008).

Sin embargo, llama la atención el porcentaje de artículos $(39,1 \%)$ que no refieren el modelo teórico, quizá esto se debe a la falta de claridad en el estudio de los factores de riesgo psicosocial laboral, tal como lo sugiere Potocka (2010) la mayoría de conceptos relativos a los factores de riesgo psicosociales, son explicados desde modelos de estrés laboral.

Según Villalobos (2007), los diferentes modelos teóricos han dado lugar a desarrollos tanto conceptuales como investigativos e instrumentales importantes, que han permitido avanzar en la comprensión de la compleja relación de los aspectos sociales, individuales y laborales, en cuanto determinan el curso de los procesos salud-enfermedad en grupos humanos. Parece haber un desarrollo más significativo en modelos que se aproximan de forma directa al estrés, y a través de este retoman los factores de riesgo bajo la denominación de estresores.

Los factores de riesgo psicosocial individual $(91,7 \%)$ e intralabral $(85,7 \%)$ son los más mencionados, mientras que los factores de riesgo extralaborales solo se mencionan en el $18,8 \%$ de los artículos. Al revisar las diferentes clasificaciones de riesgos psicosociales laborales, se encuentra que todas las organizaciones y los autores plantean como parte de sus clasificaciones los factores intralaborales, mientras que tan solo Villalobos (2007), el Observatorio Permanente de Riesgos Psicosociales (2006) y la Organización Internacio- nal del Trabajo (OIT, 2010b) consideran como parte de sus clasificaciones los factores individuales.

De esta manera, y desde las mismas clasificaciones, se observa la tendencia al estudio de los factores intra e individuales, y en esta última categoría básicamente factores sociodemográficos del trabajador. Con respecto a los factores de riesgo intralaboral más mencionados en los artículos, se encuentran control del trabajo, demandas del trabajo, y soporte social, aspectos que coinciden con el modelo teórico de demanda-control, planteado por Karasek y Theorell (1990).

En Colombia, este modelo teórico permite la comprensión y análisis de dos de los dominios planteados en el instrumento de factores de riesgo intralaboral -demandas del trabajo y control-, el cual hace parte de la batería del Ministerio de la Protección Social, validada para la población trabajadora colombiana, afiliada al Sistema de Seguridad Social en Salud. Según el Ministerio, las condiciones intralaborales son características del trabajo y de su organización que influyen en la salud y bienestar del individuo (Ministerio de la Protección Social, 2010).

En esta misma categoría de análisis se observa que los factores de riesgo extralaboral que más se mencionan en los artículos son factores relacionados con el entorno familiar, como soporte social, situación económica, composición, y relaciones familiares. Por su parte, existen otros factores que solo se mencionan en un artículo, como poca participación social, tiempo de desplazamiento, doble rol (en el caso de las mujeres) y trabajo en casa.

Varios de los riesgos psicosociales extralaborales referidos en los diferentes artículos son abordados en la batería de instrumentos para la evaluación de factores de riesgo psicosocial, donde se plantea que estas condiciones se convierten en factor de riesgo cuando las relaciones familiares son conflictivas o inexistentes, los ingresos no son suficientes para cubrir necesidades básicas, y en general cuando las condiciones no permiten el desarrollo y bienestar del trabajador.

Dada la amplia clasificación de factores psicosociales, se observa predominio del estudio de los 
factores psicosociales intralaborales, aspecto que resalta el Instituto Sindical de Trabajo, Ambiente y Salud (ISTAS, 2005). Los riesgos psicosociales laborales no son un problema individual, de personalidad, o que responda a circunstancias personales o familiares, sino que tienen su origen en las condiciones de trabajo, y específicamente en las derivadas de su organización.

En conclusión, el análisis de los artículos sobre riesgos psicosociales laborales en el periodo 2000 2010 permite evidenciar que la investigación en esta temática se encuentra en un estado exploratorio y descriptivo. Así entonces, se hace necesario realizar investigaciones desde enfoques cualitativos y mixtos, así como el uso de diseños cuasiexperimentales y experimentales que permitan profundizar en la comprensión de este fenómeno.

\section{Referencias}

Andrade, V., y Gómez, I. C. (2008). Salud laboral. Investigaciones realizadas en Colombia. Pensamiento Psicológico, 4(10) 9-25.

Barling, J., Kelloway, K., \& Iverson, R. (2003). High-Quality work, job satisfaction, and occupational injuries. Journal of Applied Psychology, 88 (2), 276-283.

Bennett, J., Lehman, W., \& Reynolds, G. (2000). Team awareness for workplace substance abuse prevention: The empirical and conceptual development of a training program. Prevention Science, 1(3) 157- 172.

Bond, F., \& Bunce, D. (2001). Job control mediates change in a work reorganization intervention for stress reduction. Journal of Occupational Health Psychology, 6(4), 290-302.

Bond, M., Punnett, L., Pyle, J., Cazeca, D., \& Cooperman, M. (2004). Gendered work conditions, health, and work outcomes. Journal of Occupational Health Psychology, 9(1), 28-45.

Bültmann, U., Kant, I., Van den Brandt, P., \& Kasl, S. (2002, febrero). Psychosocial work charac- teristics as risk factors for the onset of fatigue and psychological distress: Prospective results from the Maastricht cohort study. Psychological Medicine, 32(2), 333-345.

Camps, M., \& Chauhan, P. (Eds.). (2009). Sourcebook of paleolithic transitions: Methods, theories and interpretations. Nueva York: Springer.

Cavanaugh, M., Boswell, W., Roehling, M., \& Boudreau, J. (2000). An empirical examination of self-reported work stress among U. S. managers. Journal of Applied Psychology, 85(1), 65-74.

Day, A., \& Jreige, S. (2002). Examining type A behavior pattern to explain the relationship between job stressors and psychosocial outcomes. Journal of Occupational Health Psychology, 7(2), 109-120.

Gómez, V. (2008). Factores psicosociales del trabajo y su relación con la salud percibida y la tensión arterial: Un estudio con maestros escolares en Bogotá, Colombia. Ciencia y trabajo, 10(30), 132-137.

Guillen, C., y Guil, R. (2000). Psicología del trabajo para las relaciones laborales. Madrid: McGraw-Hill.

Instituto Sindical de Trabajo Ambiente y Salud (2005) Organización del Trabajo,Salud y Riesgos Psicosociales. España: ISTAS.

Castellà, T., Fernández, L., García, N., Lázara, M., Llorens, C., Menéndez, M., y Moncada, S. (2005). Organización del trabajo, salud y riesgos psicosociales. España: Instituto Sindical de Trabajo, Ambiente y Salud (ISTAS).Jutinico, A. (1996). Factores de riesgo psicosocial: Documento técnico. Bogotá: Seguro Social.

Johnson, J.V. \& Hall, E.M. (1988). Job strain, work place social support, and cardiovascular disease: A cross-sectional study of a random sample of Swedish working population. American Journal Public Health, 78(10), 13361342. 
Karasek, R., \& Theorell, T. (1990). Healthy work: Stress, productivity, and the reconstruction of working life. Nueva York: Basic Books.

López, E. D., Ruíz, R., y Jiménez, E. (2006). La edición de revistas científicas: Directrices, criterios y modelos de evaluación. Recuperado el 20 de marzo de 2012 de http://recyt. fecyt.es/documentos/Fecyt.pdf

Ley 1090. (2006). Reglamentación psicología. Ministerio de protección social.Recuperado de ttp://www.upb.edu.co/pls/portal/docs/PAGE /GPV2_UPB_MEDELLIN/PGV2_M030_PREGRADOS/PGV2_M030040020_PSICOLOGIA/ CODIGO_ETICO/CODIGO\%20DEONTOLOGICO\%20Y\%20BIOETICO.PDF

Melía, J. L., Nogareda, C., Duro, A., Peiró, J. M., Salanova, M., y Gracia, D. (2006). Principios comunes para la evaluación de los riesgos psicosociales en la Empresa. En J. L. Melía, C. Nogareda, M. Lahera, R. Pou, M. Salanova, A. Duro, ... F. Martinez-Loza (Eds.), Perspectivas de intervención en riesgos psicosociales. Evaluación de riesgos (pp. 13-36). Barcelona: Foment del Trabell Nacional.

Meneses, A. L., Calle, A. M., y García, M. P. (2008). Análisis bibliométrico del abordaje psicológico de la enfermedad cardiovascular de enero de 1998 a diciembre de 2007. (Trabajo de grado, Universidad El Bosque, Bogotá).

Ministerio de la Protección Social. (2008). Resolución 2646 de julio 17 de 2008. Bogotá: Ministerio de la Protección Social.

Ministerio de la Protección Social. (2010). Batería de instrumentos para la evaluación de factores de riesgo psicosocial. Bogotá: Ministerio de la Protección Social.

Ministerio del Trabajo y Asuntos Sociales de España. (s. f.). NTP 603: Riesgo psicosocial: El modelo demanda-control-apoyo social (I). Recuperado el 20 de octubre de 2010 de http://www.insht.es/InshtWeb/Contenidos/ Documentacion/FichasTecnicas/NTP/Ficheros /601a700/ntp_603.pdf
Observatorio Permanente de Riesgos Psicosociales. (2006). Guía sobre factores de riesgo psicosociales. Recuperado el 14 de marzo de 2006 de http://extranet.ugt.org/saludlaboral/ oprp/Documentos\%20Noticias/Gu\%C3\%ADa Factores_Psicosociales.pdf.

Organización Internacional del Trabajo (OIT). (2010a). Riesgos emergentes y nuevos modelos de prevención en un mundo de trabajo en transformación. Ginebra: OIT.

Organización Internacional del Trabajo (OIT). (2010b). 307a reunión. Reunión de expertos sobre la revisión de la lista de enfermedades profesionales. Recomendación núm. 194. Ginebra: OIT.

Oliver, A., Cheyne, A., Thomas, J., \& Cox, S. (2002). The effects of organizacional and individual factors on occupational accidents. Journal of Occupational and Organizacional Psychology, 75, 473-488.

Peiró, J. M. (2004). El sistema de trabajo y sus implicaciones para la prevención de los riesgos psicosociales en el trabajo. Universitas Psicológica, 3(2), 179-186.

Potocka, A. (2010). Co wiemy o psychospołecznych zagrożeniach w środowisku pracy? część i. rozważania teoretyczne. Medycyna, 61(3), 341-352.

Resolución 008430.(1993). Establecimiento de normas científicas, técnicas y administrativas para la investigación en salud. Ministerio de Salud. Recuperado de www. urosario.edu.co/.../a2/a24fb07a-f561-4fccb611-affff4374bb7.pdf

Sancho, R. 1990. Indicadores bibliométricos utilizados en la evaluación de la ciencia y la tecnología. Revisión bibliográfica. Revista Española de Documentación Científica, 13, 842-865.

Schaubroeck, J., Jones, J., \& Xie, J. (2001). Individual differences in utilizing control to cope with job demands: Effects on susceptibility to infectious disease. Journal of Applied Psychology, 86(2), 265-278 
Schaufeli, W.B \& Salanova, M.L. (2002). ¿Cómo evaluar los riesgos psicosociales en el trabajo?.Gestión Practica de Riesgos Laborales, (20), 4-9.
Villalobos, G. (2007). Diseño de un programa de vigilancia epidemiológica de factores de riesgo psicosocial en el trabajo. Bogotá: Carluma. 